\title{
Discrete layer-stripping algorithms and feasibility conditions for the 2D inverse conductivity problem
}

\author{
Andrew E Yagle \\ Department of Electrical Engineering and Computer Science, The University of Michigan, Ann \\ Arbor, MI 48109-2122, USA \\ E-mail: aey@eecs.umich.edu
}

Received 24 May 2000

\begin{abstract}
We develop a discrete layer-stripping algorithm for the 2D inverse conductivity problem. Unlike previous algorithms, this algorithm transforms the problem into a time-varying 1D Schrödinger equation inverse scattering problem, discretizes this problem and then solves the discrete problem exactly. This approach has three advantages: (i) the poor conditioning inherent in the problem is concentrated in the solution of a linear integral transform at the beginning of the problem, to which standard regularization techniques may be applied and (ii) feasibility conditions on the transformed data are obtained, satisfaction of which ensures that (iii) the solution of the discrete nonlinear inverse scattering problem is exact and stable. Other contributions include solution of discrete Schrödinger equation inverse potential problems with time-varying potentials by both layer-stripping algorithms and solution of nested systems of equations which amount to a time-varying discrete version of the Gel'fand-Levitan equation. An analytic and numerical example is supplied to demonstrate the operation of the algorithm.
\end{abstract}

\section{Introduction}

\subsection{Background}

The $2 \mathrm{D}$ inverse conductivity problem is to reconstruct the $2 \mathrm{D}$ spatially varying conductivity (or its reciprocal, resistivity) of a 2D medium from measurements of the electrical potential induced on its boundary from point current sources. Direct (zero-frequency) current (DC) is injected into the medium using a point electrode, and the resulting electrical potential is measured on the boundary of the medium. The lateral location of the point current source is varied and the experiment is repeated, so that the data consist of electrical potential as a function of both source location and measurement location. In this paper we consider the specific application of reconstructing the subsurface of the earth from measurements taken at the earth's surface, which is assumed to be a flat insulating plane over the range of the experiment.

There have been many algorithms which solve linearized versions of this problem (analogous to the Born approximation in scattering problems), such as filtered backprojection. We show below, in an analytic example, that linearization neglects significant effects in the problem. Some other approaches iterate on the forward problem to minimize an error functional, but these can encounter local minima that interfere with obtaining the correct solution. Since our approach is quite different from these, we do not discuss these approaches further here. 


\subsection{Previous layer-stripping inverse conductivity problem algorithms}

Our approach does not linearize the problem; rather it solves the full nonlinear problem. It uses layer-stripping ideas, but in a manner entirely different from previous applications of layer stripping to inverse conductivity problems. In [1-4] recursive layer-stripping algorithms were applied to the $1 \mathrm{D}$ inverse resistivity problem, and in [5] to the $2 \mathrm{D}$ inverse resistivity problem. All of these required that the number of dimensions of the medium exceed the number of dimensions of resistivity variation, e.g. a 3D medium whose conductivity varied in only two dimensions. This allowed an integral transformation first used in [6] to transform the elliptic partial differential equation inverse problem into a hyperbolic inverse problem with a time-independent scattering potential.

While this transformation requires an inverse Laplace transform, the resulting inverse scattering problem is well posed and can be solved using fast signal processing algorithms $[4,5]$. The problem of numerically computing the inverse Laplace transform is known to be ill conditioned, but there are established techniques for dealing with this problem [7]. The reader is referred to [7] for details, which we do not review here.

More recently (e.g. [8]), layer-stripping ideas have been applied to the inverse problem of reconstructing the $2 \mathrm{D}$-varying conductivity of a $2 \mathrm{D}$ medium defined on a disc, from boundary measurements of the electrical potential induced by point current sources. This is not the problem solved in this paper, but it is relevant prior work. This approach reconstructs the conductivity radially inward by defining $R(r)$ as the inverse of the Dirichlet-to-Neumann mapping at radial distance $r$, and propagating a finite-difference approximation to $R(r)$ in decreasing $r$, reconstructing the conductivity from the limiting behaviour of $R(r)$. This is analogous to the Riccati equation form of layer stripping [9], except that $R(r)$ is a current-tofield mapping and not a reflection response.

The problem with this approach is that the conductivity must be recovered from highwavenumber behaviour of $R(r)$, which is very ill posed. Analogous problems arise when using the analogous 'initial-value-theorem' method of reconstructing reflectivity in $1 \mathrm{D}$ inverse scattering [9]. Also, there is no reason to believe that the finite-difference approximation to $R(r)$ is well behaved or stable (unlike the discrete Riccati equation used in [9], which is known to be stable, since it maps the unit disc to itself). Small errors at each step can quickly cause the algorithm to diverge; this is why layer-stripping algorithms have the reputation of being unstable. We have shown in [13] that layer-stripping algorithms applied to noisy data that have been rendered feasible can be stable, confirming results we first noted in [14]. We derive an analogous feasibility result for the present problem.

\subsection{Contributions of this paper}

Our approach avoids these problems by concentrating all of the conditioning and discretization issues at the beginning of the problem. Rather than perform an ill conditioned operation at each step of the algorithm, we concentrate all of the ill conditioning into a linear transformation similar to, but not the same as, the transformation of [6] which was used in [4] and [5]. Regularization techniques can be applied to this linear transformation to improve conditioning of the overall problem. Since the transformation consists of a Fourier transform followed by an inverse Laplace transform, numerical techniques for the latter [7] may be used. The resulting algorithm is numerically stable, provided the data are feasible, and the discrete formulation allows a feasibility condition on the transformed data to be identified.

Specific contributions of this paper include the following:

(1) a new linear transformation that transforms the 2D inverse conductivity problem with 
conductivity varying in 2D into a 1D Schrödinger equation inverse potential problem with a time-varying potential;

(2) a new layer-stripping algorithm that solves the discrete form of the 1D Schrödinger equation with a time-varying scattering potential;

(3) a time-varying discrete version of the Gel'fand-Levitan integral equation that also solves this discrete time-varying 1D Schrödinger equation;

(4) a new feasibility condition for the transformed data, and a correction procedure that can be applied to infeasible (due to noise) data and

(5) a simple but illustrative analytic example that demonstrates how the algorithm operates, and that allows its numerical performance to be evaluated (since we know the actual analytic form of the solution).

\section{Basic equations and problem formulation}

The problem is defined on the half-plane $\{(x, z), z \geqslant 0\}$, where $z$ is the depth (measured downward) from the surface $z=0$ of the earth and $x$ is the lateral position in a direction parallel to the earth's surface. We assume a $2 \mathrm{D}$ earth here, although our approach can be extended to the 3D case. The algorithm given in this paper can be applied to a $3 \mathrm{D}$ problem if the medium is homogeneous in the $y$-direction and the point sources at $x=x_{0}$ are changed to line sources extending in the $y$-direction. We assume the surface of the earth is flat and is a perfect insulator, so that the normal component of current density at the boundary away from the source is zero. The goal is to reconstruct conductivity $\sigma(x, z)$ from the electrical potential $v^{\prime}(x, z=0)$ measured at the earth's surface $z=0$ and resulting from the introduction of DC through a point electrode at various locations $\left(x=x_{0}, z=0\right)$ on the earth's surface.

The basic equations are Ohm's law and Kirchhoff's current law [1-6, 8]:

$$
j(x, z)=\sigma(x, z) \nabla v^{\prime}(x, z) ; \quad \nabla \cdot j(x, z)=0
$$

where $j(x, z)$ is the (vector) current density and the boundary condition is that the normal component of $j(x, z=0)=0$ away from the source. The normalized potential $v(x, z)=$ $\sqrt{\sigma(x, z)} v^{\prime}(x, z)$ satisfies

$$
\left(\frac{\partial^{2}}{\partial x^{2}}+\frac{\partial^{2}}{\partial z^{2}}\right) v(x, z)=V(x, z) v(x, z) ; \quad V(x, z)=\frac{\nabla^{2} \sqrt{\sigma(x, z)}}{\sqrt{\sigma(x, z)}} .
$$

Note that if the medium is locally homogeneous then $V(x, z)=0$ and the normalized potential satisfies Laplace's equation $\nabla^{2} v(x, z)=0$.

\section{Transformation from elliptic to hyperbolic problem}

\subsection{Previous elliptic to hyperbolic transformation}

In [4-6] the elliptic equations (2.2), with no $x$-dependence in $V(x, z)$, were transformed into the hyperbolic equation

$$
\left(\frac{\partial^{2}}{\partial z^{2}}-\frac{\partial^{2}}{\partial t^{2}}\right) u(z, t)=V(z) u(z, t)
$$

using the Fourier-inverse-Laplace transformation (see [4-6] for details)

$$
u(z, t)=\mathcal{L}_{k \rightarrow t}^{-1}\left\{k \mathcal{F}_{x \rightarrow k}\{v(x, z)\}\right\}
$$

which can be effected by solving the integral equation

$$
v(x, z)=-\frac{1}{\pi} \int_{0}^{\infty} \log \sqrt{t^{2}+x^{2}} u(z, t) \mathrm{d} t .
$$


This requires analytic continuation of the Fourier transform of $v(x, z)$ from the positive real axis to the entire right half of the complex $k$-plane. Proofs that this analytic continuation can be performed were given in [3-5]. $u(z, t)$ was causal due to the inverse Laplace transform and the analyticity; the extra $k$ was used to produce an impulse in the boundary condition. The integral equation (3.2) had an insightful physical interpretation of writing the potential as the superposition of potentials due to a continuous distribution of fictitious image potentials distributed along a fictitious depth $t$ (see [3-5]).

This transformation cannot be used directly on the present problem, for two reasons: (i) $V(x, z)$ now depends on $x$ and (ii) $v(x, z)$ is no longer an even function in $x$ (see section 1.2). Unlike (3.3) there are real and imaginary parts that must be treated separately. However, insightful physical interpretation of the transformation is still possible.

At first glance it seems that the $x$-dependence present in $V(x, z)$ should not cause any difficulties, since the Fourier transform converts the product $V(x, z) v(x, z)$ in (2.2) into a convolution and the inverse Laplace transform converts the convolution back into a product. However, there is a complication: previously, the lack of $x$-dependence in $V(z)$ implied that $v(x, z)$ was symmetric in $x$ about the origin $x=0$ where the impulsive point current source was located. Since the Fourier transform of a real and even function is real and even, the Fourier transform $\hat{v}(z, k)$ of $v(x, z)$ was real and even in $k$. Then we had, in this previous work,

$$
\begin{gathered}
v(x, z)=\mathcal{F}_{k \rightarrow x}^{-1} \mathcal{L}_{t \rightarrow k}\left\{\int_{0}^{t} u\left(z, t^{\prime}\right) \mathrm{d} t^{\prime}\right\}=\frac{2}{2 \pi} \int_{0}^{\infty} \operatorname{Re}\left[\int_{0}^{\infty} \int_{0}^{t} u\left(z, t^{\prime}\right) \mathrm{e}^{-k t} \mathrm{e}^{\mathrm{i} k x} \mathrm{~d} t^{\prime} \mathrm{d} t\right] \mathrm{d} k \\
=\frac{1}{\pi} \int_{0}^{\infty} \int_{0}^{t} \operatorname{Re}\left[\frac{u\left(z, t^{\prime}\right)}{t-\mathrm{i} x}\right] \mathrm{d} t^{\prime} \mathrm{d} t=-\frac{1}{\pi} \int_{0}^{\infty} \log \sqrt{t^{2}+x^{2}} u(z, t) \mathrm{d} t
\end{gathered}
$$

where integration by parts has been used to obtain the final equality in (3.4). Note the factor of $k$ in (3.2) has become the integration over $\mathrm{d} t^{\prime}$ in (3.4). The Laplace transform is only defined for $\operatorname{Re}[k]>0$ and we only needed it there.

However, $v(x, z)$ is not symmetric in $x$, since $V(x, z)$ depends on $x$. So the Fourier transforms $\hat{v}(z, k)$ and $\hat{V}(z, k)=\mathcal{F}_{x \rightarrow k}\{V(x, z)\}$ and their inverse Laplace transforms are now complex.

\subsection{New elliptic to hyperbolic transformation}

We can solve both of these problems by doing the following: (i) omitting the factor of $k$ from (3.2), which eliminates the integral over $t^{\prime}$ in (3.4) and ensures that the product $V(x, z) v(x, z)$ maps to the product $U(z, t) u(z, t)$ of the transformed quantities, and (ii) treating the real and imaginary parts of $\hat{v}(z, k)$ and $\hat{V}(z, k)$ separately and making use of conjugate symmetry. Since we know $v(x, z)$ is real, $\operatorname{Re}[\hat{v}(z, k)]$ is an even function in $k$ and $\operatorname{Im}[\hat{v}(z, k)]$ is an odd function in $k$. Using this allows us to write

$$
\begin{aligned}
v(x, z)=\mathcal{F}_{k \rightarrow x}^{-1} & \left\{u(z, t) \int_{0}^{\infty} \mathrm{e}^{-k t} \mathrm{~d} t\right\} \\
& =\frac{2}{2 \pi} \int_{0}^{\infty} \operatorname{Re}\left[\int_{0}^{\infty} u(z, t) \mathrm{e}^{-k t} \mathrm{e}^{\mathrm{i} k x} \mathrm{~d} t\right] \mathrm{d} k=\frac{1}{\pi} \int_{0}^{\infty} \operatorname{Re}\left[\frac{u(z, t)}{t-\mathrm{i} x}\right] \mathrm{d} t \\
& =\frac{1}{\pi} \int_{0}^{\infty} \operatorname{Re}[u(z, t)]\left(t /\left(t^{2}+x^{2}\right)\right) \mathrm{d} t-\frac{1}{\pi} \int_{0}^{\infty} \operatorname{Im}[u(z, t)]\left(x /\left(t^{2}+x^{2}\right)\right) \mathrm{d} t .
\end{aligned}
$$

The point here is that by treating the real and imaginary parts of $\hat{v}(z, k)$ separately, we can once again restrict attention to $\operatorname{Re}[k]>0$, where the Laplace transform is defined, and derive 
the kernel for the transformation (3.2).

The complex integral equation (3.5) can be split into two real equations by writing

$$
\begin{aligned}
& v(x, z)+v(-x, z)=\frac{2}{\pi} \int_{0}^{\infty} \operatorname{Re}[u(z, t)]\left(t /\left(t^{2}+x^{2}\right)\right) \mathrm{d} t \\
& v(x, z)-v(-x, z)=-\frac{2}{\pi} \int_{0}^{\infty} \operatorname{Im}[u(z, t)]\left(x /\left(t^{2}+x^{2}\right)\right) \mathrm{d} t .
\end{aligned}
$$

Now solve (3.6a) and (3.6b) separately to obtain $\operatorname{Re}[u(z, t)]$ and $\operatorname{Im}[u(z, t)]$.

\subsection{Interpretation of new elliptic to hyperbolic transformation}

Note the following about the transformation (3.5):

(1) If $v(x, z)$ is an even function of $x$, then $\hat{v}(z, k)$ is real and (3.5) reduces to the transformation (3.4) used in [4-6].

(2) The kernels in (3.5) show the transformation represents the actual response as a superposition of responses to fictitious image sources in a fictitious medium with $z$ replaced by $t$. See [5] for more details on this type of physical interpretation of this type of transformation.

(3) Solution of the integral equation is thus a $2 \mathrm{D}$ deconvolution problem. This shows how the inverse conductivity problem can be ill conditioned. It is well known that the medium is in effect being probed by evanescent (exponentially decaying) waves, instead of the travelling waves used in scattering problems, so this is not surprising.

(4) Instead of solving (3.6), we can simply implement (3.2) (without the factor $k$ ). This requires numerical computation of an inverse Laplace transform, but this problem is well understood and techniques for computation and regularization are known [7].

Two simple examples of the transformation (3.5) are now presented. It is clear that (3.5) maps an impulse $\delta(x)$ to $\delta(t)$, and vice versa. To determine what function maps to a unit step (Heaviside) function $1(t)$, note that

$$
\begin{gathered}
\mathcal{F}_{k \rightarrow x}^{-1} \mathcal{L}_{t \rightarrow k}\{1(t)\}=\mathcal{F}^{-1}\{1 / k\}=\frac{1}{2 \pi} \int_{-\infty}^{\infty} \frac{\cos (k x)}{k} \mathrm{~d} k+\frac{\mathrm{i}}{2 \pi} \int_{-\infty}^{\infty} \frac{\sin (k x)}{k} \mathrm{~d} k \\
=\frac{2 \mathrm{i}}{2 \pi} \int_{0}^{\infty} \frac{\sin (k x)}{k} \mathrm{~d} k=\frac{2 \mathrm{i}}{2 \pi} \frac{\pi}{2} \operatorname{sgn}(x)=\frac{\mathrm{i}}{2} \operatorname{sgn}(x)
\end{gathered}
$$

where $\operatorname{sgn}(x)$ is the sign \pm 1 of $x$. The first integral is zero is the sense of the Cauchy principal value. Heuristically, these results can be linked (in a very nonrigorous but illustrative way) by observing $\delta(t)=(\mathrm{d} / \mathrm{d} t) 1(t)=(\mathrm{d} / \mathrm{d}(\mathrm{i} x))(\mathrm{i} / 2) \operatorname{sgn}(x)=\delta(x)$, since the transformation performs an analytic continuation from the real axis to the imaginary axis, i.e. ' $t=\mathrm{i} x$ '.

For a delayed step function, (3.7) becomes

$\mathcal{F}^{-1}\left\{\frac{\mathrm{e}^{-k t_{0}}}{k}\right\}=-\frac{1}{\pi} \int_{0}^{\infty} \frac{\sinh \left(k t_{0}\right) \cos (k x)}{k} \mathrm{~d} k+\frac{\mathrm{i}}{\pi} \int_{0}^{\infty} \frac{\cosh \left(k t_{0}\right) \sin (k x)}{k} \mathrm{~d} k$.

Again two integrals are zero in the sense of the Cauchy principal value. Note that both integrands in (3.8) are finite at $k=0$, and the integrals converge for $t_{0} \geqslant 0$.

\subsection{Synthesis of delayed impulsive sources}

The mapping (3.5) maps solutions of the elliptic equations (2.2) to solutions of a hyperbolic equation (3.1) in which $V(z)$ is replaced by the time-varying scattering potential $U(z, t)$. We show how to solve the $1 \mathrm{D}$ inverse scattering problem for this equation in section 4 below. 
However, we still have to find boundary conditions for (2.2) that map to causal boundary conditions for (3.1). The causality in time $t$ of $u(z=0, t)$ in (3.1) is necessary for solution of the inverse scattering problem, since the causal signal will propagate through the hyperbolic system (3.1). We can do this as follows.

In section 4 we formulate the inverse scattering problem as using a probing impulse $\delta\left(t-t_{0}\right)$ to probe the medium. This is done to match previous work [9-15] on this problem. Actually, section 4 (equations (4.3) and (4.5) in particular) makes it clear that the impulse is not necessary. Accordingly, we may omit the factor of $k$ from the previously used transformation (3.2). However, it is necessary to obtain a family of solutions $u\left(z=0, t ; t_{0}\right)=0$ for $t<t_{0}$, for various $t_{0} \geqslant 0$, from the measured electrical potentials $v(x, z=0)$ resulting from point current sources along the $x$-axis at the surface $z=0$.

Let $v\left(x, z=0 ; x_{0}\right)$ be the potential resulting from an impulsive current source at $x=x_{0}$. Then by linearity the linear combination of solutions $\int_{-\infty}^{\infty} h\left(x_{0}\right) v\left(x, z=0 ; x_{0}\right) \mathrm{d} x_{0}$ for any weights $h\left(x_{0}\right)$ satisfies (2.2); this is the response of the medium to a superposition of impulsive point current sources weighted by $h\left(x_{0}\right)$. We need to choose the weights $h\left(x_{0}\right)$ so that the transformation from $v\left(x, z=0 ; x_{0}\right)$ to $u\left(z=0, t ; t_{0}\right)$ results in $u\left(z=0, t ; t_{0}\right)=0$ for $t<t_{0}$.

More specifically, we need to determine weights $h\left(x_{0} ; t_{0}\right)$ so that

$$
\mathcal{L}_{k \rightarrow t}^{-1} \mathcal{F}_{x \rightarrow k} \int h\left(x_{0} ; t_{0}\right) v\left(x, z=0 ; x_{0}\right) \mathrm{d} x_{0}=0, \quad 0<t<t_{0} .
$$

This becomes the following integral equation, which is to be solved for the weights $h\left(x_{0}, ; t_{0}\right)$ separately for each $t_{0}$ :

$$
\begin{aligned}
& \int_{-\infty}^{\infty} \mathrm{d} x_{0}\left(\int_{-\infty}^{\infty} G(x, t) v\left(x, z=0 ; x_{0}\right) \mathrm{d} x\right) h\left(x_{0} ; t_{0}\right)=0, \\
& 0<t<t_{0} ; \quad G(x, t)=\mathcal{L}_{k \rightarrow t}^{-1}\left\{\mathrm{e}^{-\mathrm{i} k x}\right\} .
\end{aligned}
$$

Note that the inner part of (3.10) implements the inverse of the transformation (3.5), in order to force $u(z=0, t)=0$ for $t<t_{0}$. After the weights $h\left(x_{0} ; t_{0}\right)$ have been determined, we then solve (3.5) to determine the nonzero values of $u\left(z=0, t ; t_{0}\right)$.

In practice, $x, t, x_{0}, t_{0}$ are all discretized to integer multiples of some small $\Delta$, so (3.5) and (3.10) become linear systems of equations. In this context, it is easy to see why we need the transformation in both the forward (3.5) and inverse (3.10) directions. The procedure amounts to solving

$$
\left[\begin{array}{ll}
A_{11} & A_{12} \\
A_{21} & A_{22}
\end{array}\right]\left[\begin{array}{l}
x \\
0
\end{array}\right]=\left[\begin{array}{l}
b \\
y
\end{array}\right] .
$$

Portions of both vectors are known ( $b$ and 0$)$ and unknown $(x$ and $y$ ), so it is necessary to implement both forward and inverse linear transformations.

Heuristically, the effects of the forward (3.5) and inverse (3.8) transformations are to perform analytic continuations of the functions being transformed from the real axis to the imaginary axis. Keeping this in mind can greatly simplify matters on occasion (see the example in section 5).

\subsection{Summary of elliptic-to-hyperbolic mapping procedure}

(1) Measure the electrical potential $v\left(x, z=0 ; x_{0}\right)$ at the surface $z=0$ resulting from an impulsive point current source on the surface at lateral location $x=x_{0}$.

(2) For each $t_{0} \geqslant 0$, solve (3.10) for the weights $h\left(x_{0} ; t_{0}\right)$ and define $v(x, z=0)=$ $\int_{-\infty}^{\infty} h\left(x_{0} ; t_{0}\right) v\left(x, z=0 ; x_{0}\right) \mathrm{d} x_{0}$. In practice we will only have $v\left(x, z=0 ; x_{0}\right)$ for a finite range of $x_{0}$. 
(3) Solve separately the two equations (3.6), or equivalently (3.5), for $u(z=0, t)$. Do this separately for each $t_{0} \geqslant 0 . u(z=0, t)$ is used to initialize the layer-stripping algorithm which consists of (4.3) and (4.5) below. Alternatively, compute the transformation (3.2) (without the factor $k$ ).

(4) From the boundary condition stated below (2.1), we know that the gradient of $v(x, z=$ $\left.0 ; x_{0}\right)$ in the vertical $(z-)$ direction is zero at $z=0$. This is needed to initialize the discrete Schrödinger equation (4.3) below.

\section{Time-varying Schrödinger equation inverse scattering problems}

\subsection{Formulation of the time-varying inverse scattering problem}

We now apply the transformation specified by (3.5) to (2.2) to obtain the hyperbolic equation with time-varying potential (compare with (3.1)):

$$
\left(\frac{\partial^{2}}{\partial z^{2}}-\frac{\partial^{2}}{\partial t^{2}}\right) u(z, t)=U(z, t) u(z, t) .
$$

This is technically a time-varying plasma wave equation, which is the inverse Fourier transform of a Schrödinger equation in the time-invariant case. For convenience we will refer to the entirely time-domain equation (4.1) as a time-varying Schrödinger equation, although this is not strictly accurate.

The boundary conditions are a radiation condition for large $z$ and

$u(z=0, t)=\delta\left(t-t_{0}\right)+r\left(t, t_{0}\right), \quad t>t_{0} ; \quad u(z=0, t)=0, \quad t<t_{0}$.

The goal is to reconstruct the time-varying potential $U(z, t)$ from the response $r\left(t, t_{0}\right)$. In practice, it will become clear that the impulse in (4.2) is in fact unnecessary, but we include it to facilitate comparison with the results of [9-15].

Discretizing (4.1) and setting $z=n \Delta, t=i \Delta$ and $t_{0}=j \Delta$ gives

$u_{n+1}(i, j)+u_{n-1}(i, j)-u_{n}(i+1, j)-u_{n}(i-1, j)=V_{n}(i) u_{n-1}(i, j)$

where $u_{n}(i, j)=u(z=n \Delta, t=i \Delta)$ resulting from an impulse at $t_{0}=j \Delta$ and $V_{n}(i)=U(z=n \Delta, t=i \Delta) \Delta^{2}$. The boundary conditions are

$$
u_{0}(i, j)=\delta(i-j)+2 r_{i, j} ; \quad u_{N}(i, j)=x_{i, j}
$$

where $r_{i, j}=r\left(t=i \Delta, t_{0}=j \Delta\right) \Delta$ and the impulse is now discrete.

Note that $r_{i, j}$ is the reflection response at time $i$ to an impulse at time $j$, and $x_{i, j}$ is the transmission response at time $i$ to an impulse at time $j$. Also note that the wavefield $u_{n}(i, j)$ is the sum of downgoing and upgoing waves, and that in practice the discrete impulse can easily be lumped in with $r_{i, j}$.

The impulse at the surface $n=0$ at time $j$ will propagate at unit wavespeed through the medium and be reflected by the scattering potential $V_{n}(i)$. If $V_{n}(i)=0$ then there is no scattering at depth $n$ and the scattered field simply passes through depth $n$ without further scattering. If $V_{n}(i) \neq 0$ then scattering will occur, and some of this will make it back to the surface $n=0$ at time $i$ as the reflection response $r_{i, j}$. If $V_{n}(i)$ does not depend on time, the medium is time invariant and $r_{i, j}=r_{i-j}$. Since $i \geqslant j \geqslant 0$, we may without loss of generality set $V_{n}(-i)=V_{n}^{*}(i)$; since the impulse cannot reach depth $n>0$ at time $i=0$ this has no effect on the physical problem, but it simplifies the mathematics below. 


\subsection{Layer stripping solution of time-varying inverse scattering problem}

Causality shows $u_{n}(i, j)=0$ if $i<n+j$. Setting $i=n-1+j$ in (4.3) yields

$$
V_{n}(n-1+j)=-\frac{u_{n}(n+j, j)-u_{n-1}(n-1+j, j)}{u_{n-1}(n-1+j, j)},
$$

which states that the difference of the first nonzero values of the wavefield at depths $n$ and $n-1$ determines the potential at depth $n$ at time $n-1+j$.

For an inherently discrete problem, or for a discretized continuous problem which does not include an impulse in the boundary condition, (4.5) should be used. However, for a discretized continuous problem which does include an impulse in the boundary condition, the expression in (4.5) should be doubled. The reason for this is derived in section 5. A similar distinction was obtained in [9].

Now suppose we have $r_{i, j}$ for all $i, j \geqslant 0$. Then we can propagate (4.3) and (4.5) together, reconstructing $V_{n}(i)$ from $u_{n}(i, j)$ and $u_{n-1}(i, j)$ using (4.5), and then inserting the reconstructed $V_{n}(i)$ into (4.3) to obtain $u_{n+1}(i, j)$ etc. This recursive reconstruction is the hallmark of a layer-stripping algorithm. However, this particular algorithm applies to a time-varying scattering medium, done before only in [10]. [10] considered a time-varying two-component wave system, while this paper considers time-varying Schrödinger equations, and applies the results to the inverse conductivity problem.

Note the following about this algorithm:

(1) We need to propagate separate copies of (4.3) for each impulse time $j$, in order to reconstruct $V_{n}(i)$ for all $i \geqslant n-1$ and then broadcast the $V_{n}(i)$ to the separate copies of (4.3) at each recursion.

(2) This algorithm is essentially a time-varying 'split' algorithm. The split versions of the Levinson and Schur algorithms are well known algorithms in signal processing, and are known to be numerically stable in the time-invariant case. This is a new generalization of the split algorithms, but there is reason to believe that these algorithms will also be stable.

(3) Discretization to $N$ points in space $z$ and time $t$ will require $\mathrm{O}\left(N^{3}\right)$ multiplications and additions to reconstruct the potential.

(4) For a time-invariant medium we require only one copy of (4.3), since $r_{i, j}=r_{i-j}$ and $u_{n}(i, j)=u_{n}(i-j)$. Then $\mathrm{O}\left(N^{2}\right)$ operations are needed.

(5) In the time-invariant case and in the continuous limit $\Delta \rightarrow 0,(4.5)$ corresponds to the formula $V(z)=-2(\mathrm{~d} / \mathrm{d} z) u(z, z)$ [9], since the impulse cancels in the numerator but dominates the denominator. In the time-varying case a similar formula holds (see section 5).

\subsection{Discrete time-varying Gel'fand-Levitan integral equation}

As an alternative to the layer-stripping algorithm, we can solve the inverse scattering problem by solving a nested set of non-Toeplitz systems of equations. We show that, in the continuous limit for a real and time-invariant medium, this reduces to the Gel'fand-Levitan integral equation of inverse scattering [9]. It also results in a feasibility condition for the reflection response $r_{i, j}$. Although this requires more computation than the layer-stripping algorithm, this approach is guaranteed to be stable if Gaussian elimination with pivoting is used.

Recall that $r_{i, j}$ is the reflection response at time $i$ to an impulse which is at the surface $n=0$ at time $j$. Also recall that we define $V_{n}(-i)=V_{n}^{*}(i)$ for negative times, which does not affect the physical problem. Now reverse time by replacing $i$ with $-i$ and $j$ with $-j$. A slight modification of an argument given in [10] shows that

$$
r_{-i,-j}=r_{j, i}^{*} \text {. }
$$


To see this, consider the response observed at a fixed time $i$ due to a series of experiments initiated at various times $j<i$, and also consider the responses observed at a series of times $i$ due to a single experiment initiated at time $j$. All primary and multiple reflections occur at times measured backward from $i$ in the first case, and forward from $j$ in the second case. Furthermore, if time is reversed and negative times $i, j<0$ are considered, the conjugation of $V_{n}(-i)=V_{n}^{*}(i)$ will clearly conjugate the reflection response $r_{-i,-j}$. Combining these ideas gives (4.6).

Now let $h_{n}(i, k)$ be the impulse response (Green function) of the medium described by (4.3). $h_{n}(i, k)$ relates the wavefield $u_{n}(i, j)$ at depth $n$ and time $i$ to the wavefield $u_{0}(k, j)$ at the surface $n=0$ at time $k$, where the impulse is at the surface at time $j\left(h_{n}(i, k)\right.$ does not depend on $j$ since $h_{n}(i, k)$ is a property of the medium itself, not of its probing). $h_{n}(i, k)$ satisfies (4.3) with the initial condition $h_{0}(i, k)=\delta(i-k)$. The structure of $(4.3)$ and $V_{n}(-i)=V_{n}^{*}(i)$ show that $h_{n}(i, k)$ is Hermitian in time: $h_{n}(-i,-k)=h_{n}^{*}(i, k)$ (note $h_{n}(i, k)$ is noncausal with support $|i-k| \leqslant n)$. Using the boundary conditions (4.4) yields

$$
u_{N}(i, j)=x_{i, j}=\sum_{k=i-N}^{i+N} h_{N}(i, k)\left(\delta(k-j)+2 r_{k, j}\right) .
$$

Now reverse time by replacing $i$ with $-i, j$ with $-j$ and $k$ with $-k$. Adding (4.7) to the conjugate of its time reversal and using (4.6) yields

$$
\begin{aligned}
x_{i, j}+x_{-i,-j}^{*} & =\sum_{k=i-N}^{i+N} h_{N}(i, k)\left(2 \delta(k-j)+2 r_{k, j}+2 r_{-k,-j}^{*}\right) \\
& =\sum_{k=i-N}^{i+N} h_{N}(i, k) 2\left(\delta(k-j)+r_{k, j}+r_{j, k}\right)
\end{aligned}
$$

which can be written as the system of equations

$$
\left[\begin{array}{ccccc}
1 & r_{i-N+1, i-N} & r_{i-N+2, i-N} & \cdots & r_{i+N, i-N} \\
r_{i-N+1, i-N} & 1 & r_{i-N+2, i-N+1} & \cdots & r_{i+N, i-N+1} \\
r_{i-N+2, i-N} & r_{i-N+2, i-N+1} & 1 & \cdots & r_{i+N, i-N+2} \\
\vdots & \vdots & \vdots & \vdots & \vdots \\
r_{i+N, i-N} & r_{i+N, i-N+1} & r_{i+N, i-N+2} & \cdots & 1
\end{array}\right]\left[\begin{array}{c}
h_{N}(i, i-N) \\
h_{N}(i, i-N+1) \\
h_{N}(i, i-N+2) \\
\vdots \\
h_{N}(i, i+N)
\end{array}\right]=\left[\begin{array}{c}
t \\
0 \\
0 \\
\vdots \\
0
\end{array}\right]
$$

where $2 t=x_{i, i-N}+x_{-i,-i+N}^{*}$ is defined from the unknown first value $x_{i, i-N}$ of the transmission response. However, this unknown is compensated by the impulse $\delta(i-k)$ known to be present in $h_{N}(i, i \pm N)$.

In (4.9) we can replace $N=$ total number of layers with any $n$ and (4.9) will still hold by causality. We can thus solve the time-varying inverse scattering problem by solving the nested systems of equations (4.9) and then using

$$
V_{n}(n-1+j)=\frac{h_{n}(n+j, j)-h_{n-1}(n-1+j, j)}{h_{n-1}(n-1+j, j)}
$$

which can be derived in the same way as (4.5). Note that $h_{n}(i, j)=0$ for $|i-j|>n$ while $u_{n}(i, j)=0$ for $i-j<n$; this is the sign difference between (4.5) and (4.10).

To show that (4.8) is a generalized discrete time-varying Gel'fand-Levitan integral equation, consider a time-invariant medium in which $V_{n}(i)$ is real and does not depend on time $i$. Then $h_{n}(i, j)=h_{n}(i-j), r_{i, j}=r_{i-j}$ and $x_{i, j}=x_{i-j}$. Since $h_{n}(i, j)=h_{n}(-i,-j)$, $h_{n}(i)$ is an even function and we can write $h_{n}(i)=\tilde{h}_{n}(i)+\tilde{h}_{n}(-i)$, where $\tilde{h}_{n}(i)=0$ for $i<0$ 
(recall $h_{n}(i)=0$ for $\left.|i|>n\right)$. Inserting these into (4.8) gives

$$
\begin{aligned}
x_{i}+x_{-i}^{*}= & \sum_{k=i-N}^{i+N} h_{N}(i-k)\left(2 \delta(k)+2 r_{k}+2 r_{-k}\right)=2 \sum_{k=-N}^{N} h_{N}(k)\left(\delta(i-k)+r_{|i-k|}\right) \\
& =2 h_{N}(i)+2 \sum_{k=-N}^{N}\left(\tilde{h}_{N}(k)+\tilde{h}_{N}(-k)\right) r_{|i-k|} \\
& =2 h_{N}(i)+2 \sum_{k=0}^{N} \tilde{h}_{N}(k)\left(r_{|i-k|}+r_{i+k}\right)
\end{aligned}
$$

which has the Toeplitz-plus-Hankel kernel of the Gel'fand-Levitan equation (note $r_{i}$ is causal). The left side $x_{i}=0$ for $i<N, h_{N}(i)=\tilde{h}_{N}(i)$ for $i>0$ and noting that $h_{N}(i)$ contains an impulse we obtain for $0<i<N$ the Gel'fand-Levitan equation in the continuous limit $\Delta \rightarrow 0$. This justifies calling (4.9) a discrete time-varying Gel'fand-Levitan equation generalization.

We note that the Toeplitz-plus-Hankel kernel of the Gel'fand-Levitan equation is in fact a consequence of the time symmetry of the Green function $h_{n}(i)$. The equation can be rewritten with a purely Toeplitz kernel and a time-symmetric unknown. This equivalence also appears in two-sided linear prediction [11].

\subsection{Feasibility condition}

We now obtain a feasibility condition for the reflection response $r_{i, j}$. This is the same feasibility condition as obtained in [10] for a time-varying two-component wave system; here we show that it also applies for a time-varying Schrödinger equation. However, the derivation is entirely different.

Consider two scattering experiments: one in which the medium is probed from its top layer $n=0$, and one in which it is probed from its bottom layer $n=N$. The boundary conditions defining the two experiments are

$$
\begin{array}{ll}
u_{0}(i, j)=\delta(i-j)+2 r_{i, j} ; & u_{N}(i, j)=x_{i, j} \\
u_{N}(i, j)=\delta(i-j)+r_{i, j}^{\prime} ; & u_{0}(i, j)=2 x_{i, j}^{\prime}
\end{array}
$$

In the first experiment (which we have been considering all along), an impulsive probing from the top results in reflection response $r_{i, j}$ and transmission response $x_{i, j}$. In the second experiment, an impulsive probing from the bottom results in reflection response $r_{i, j}^{\prime}$ coming out of the bottom and transmission response $x_{i, j}^{\prime}$ at the top, which is promptly reflected back into the medium due to the perfect reflector at $n=0$. We have argued above that $r_{i, j}=r_{-j,-i}^{*}$, and a similar argument shows $x_{i, j}^{*}=x_{-j,-i}^{\prime}[10]$. Note the wavefield $u_{n}(i, j)$ is the sum of the downgoing and updoing waves.

We now argue that $\sum_{k} r_{i, k}^{\prime} x_{-k,-j}=x_{i, j}$. To see this, note from (4.12a) that a probing impulse at $n=0$ produces a transmission response $x_{i, j}$. Reversing time, a probing signal $x_{-i,-j}$ at $n=N$ produces an impulse $\delta(i-j)$ at $n=0$, which is promptly reflected back into the medium and produces a transmission response $x_{i, j}$ at $n=N$. Since the medium is linear (although not time-invariant), and the impulse reflection response at $n=N$ is $r_{i, j}^{\prime}$, we have $\sum_{k} r_{i, k}^{\prime} x_{-k,-j}=x_{i, j}$.

Now add (4.12a) to its time reversal, and convolve (4.12b) with $x_{-i,-j}$ :

$$
\begin{aligned}
& u_{0}(i, j)=2 \delta(i-j)+2 r_{i, j}+2 r_{-i,-j} ; \quad u_{N}(i, j)=x_{i, j}+x_{-i,-j} \\
& u_{0}(i, j)=2 \sum_{k} x_{i, k}^{\prime} x_{-k,-j}=2 \sum_{k} x_{-k,-i}^{*} x_{-k,-j}
\end{aligned}
$$


$u_{N}(i, j)=\sum_{k}\left(\delta(i-k)+r_{i, k}^{\prime}\right) x_{-k,-j}=x_{-i,-j}+x_{i, j}$

where we have used the arguments above. Since the wavefields at $n=N$ in $(4.13 a)$ and (4.13b) are identical, the wavefields at $n=0$ must also be identical, and

$$
\delta(i-j)+r_{i, j}+r_{j, i}^{*}=\sum_{k} x_{k, i}^{*} x_{k, j}
$$

where we have reversed time by replacing $i$ with $-i$, etc and used (4.6).

Equation (4.14) is the feasibility condition. It shows that the system matrix in the generalized discrete Gel'fand-Levitan equation is positive semidefinite, and its Cholesky factor is the matrix composed of elements $x_{i, j}$. This is a generalization to time-varying media of [12], in which it was shown that for a time-invariant medium the two-sided free surface reflection response is the autocorrelation of the transmission response.

The significance of (4.14) is that the reflection responses $r_{i, j}$ are such that the matrix in (4.9) is positive semidefinite. If these responses are noisy to the point where this matrix is not positive semidefinite, then the data are infeasible, and neither layer stripping nor any other method will (or should) work. Note that the transformation discussed in section 3 could easily render data infeasible, in which case layer stripping would not work. An appropriate correction procedure would be to adjust negative eigenvalues to slightly greater than zero; this is the minimum (in the Hilbert-Schmidt norm) perturbation needed to produce feasible $r_{i, j}$ on which layer stripping will work.

Although these results reduce to previously known results for time-invariant media, the approach taken here still gives valuable insights into scattering. The simplification $r_{i, j}=r_{i-j}$ shows why Toeplitz matrices arise in the inverse scattering problem. The connection between two-sided linear prediction and the two forms of the Gel'fand-Levitan equation (Toeplitz-plusHankel kernel versus Toeplitz kernel and symmetric unknown vector) seems to be new. The approach taken to deriving (4.14) differs dramatically from either [10] or [12] and lends new insight into this result.

\section{Simple illustrative analytical and numerical example}

\subsection{Overview}

We present a simple example that illustrates the essential points of the procedure given in this paper. It is not intended to be realistic. It is simple enough that explicit analytic expressions are known for most of the variables. Such an example has three attractive features:

(1) Analytic expressions can be traced through the procedure, illustrating precisely the computations at each step, and allowing the reader to verify the operation of the procedure.

(2) Explicit analytic expressions are known for the forward problem (the electrical potentials $\left.v\left(x, z=0 ; x_{0}\right)\right)$ and the quantity to be reconstructed (conductivity $\sigma(x, z)$ and potential $V(x, z))$. Hence the accuracy of a numerical (computer) implementation of the algorithm can be assessed explicitly.

(3) This approach satisfies one of the 'rules' of inverse problem analysis: the method used to solve the forward problem (analytic result) differs from the method used to solve the inverse problem (numerical implementation).

\subsection{Problem formulation for the example}

The conductivity $\sigma(x, z)=\left(z^{2}+6 z+9\right) / x^{2}$. Note the singularity at $x=0$, corresponding to an ideal conductor along the vertical $x=0$ axis. The potential is easily computed using (2.2) 
to be $V(x, z)=2 / x^{2}$. Note that the lack of $z$-dependence in $V(x, z)$ does not imply a vertically homogeneous medium; as long as $V(x, z) \neq 0$ the medium will affect the electrical potential (2.2).

By placing current sources at various locations, we measure at the surface $z=0$ the following family of electrical potentials:

$$
\begin{gathered}
v\left(x, z=0 ; t_{0}\right)=\mathcal{F}_{k \rightarrow x}^{-1}\left\{\mathrm{e}^{-k t_{0}}\right\}-\frac{1}{\pi x} \int_{0}^{\infty} \frac{\cosh \left(k t_{0}\right) \sin (k x)}{k} \mathrm{~d} k \\
-\frac{\mathrm{i}}{\pi x} \int_{0}^{\infty} \frac{\sinh \left(k t_{0}\right) \cos (k x)}{k} \mathrm{~d} k
\end{gathered}
$$

(we skip the solution of (3.8) for the weights $h\left(x_{0}, z_{0}\right)$ ). The goal is to reconstruct the (treated as unknown) $\sigma(x, z)$ from the known $v\left(x, z=0 ; t_{0}\right)$.

Note that $v\left(x, z=0 ; t_{0}\right)$ is complex; in practice this could easily happen by taking one solution as the real part and another as the imaginary part-by linearity any linear combination of the solutions is also a solution. The complex $v\left(x, z=0 ; t_{0}\right)$ is necessary to obtain analytic expressions for all quantities arising in the procedure. However, for $t_{0}=0$ we have, following reasoning similar to that used in (3.7),

$$
v\left(x, z=0, ; t_{0}=0\right)=\delta(x)-\frac{1}{2|x|}
$$

which is entirely real valued.

\subsection{Analytic elliptic-to-hyperbolic transformation}

Applying the transformation directly to (5.1) results in

$$
u\left(z=0, t ; t_{0}\right)=\mathcal{L}_{k \rightarrow t}^{-1} \mathcal{F}_{x \rightarrow k}\left\{v\left(x, z=0 ; t_{0}\right)\right\}=\delta\left(t-t_{0}\right)-\frac{1}{t} 1\left(t-t_{0}\right),
$$

since this simply undoes the transformations used to create (5.1) (see (3.8)). It is evident that (5.3) has the form of the boundary condition (4.2) for a time-varying 1D inverse scattering problem.

The case $t_{0}=0$ is worth noting separately. Since we have seen in (5.2) that $v(x, z=$ $\left.0 ; t_{0}=0\right)$ is real, we may apply (3.6a) here. Applying (3.6a) to the nonimpulsive term in (5.3) gives

$v(x, z=0)+v(-x, z=0)=\frac{2}{\pi} \int_{0}^{\infty}-\frac{1}{t} \frac{t}{t^{2}+x^{2}} \mathrm{~d} t=-\frac{2}{\pi} \frac{\pi}{2|x|}=-\frac{1}{|x|}$,

which agrees with (5.2)

For the potential $V(x, z)$, we have

$$
U(z, t)=\mathcal{L}^{-1} \mathcal{F}\left\{2 / x^{2}\right\}=-2 / t^{2} .
$$

Heuristically, this result can be understood by noting that the effect of the transformation is to analytically extend the function $2 / x^{2}$ from the real to the imaginary axis ' $x=\mathrm{i} t$ ' -we simply replace $x^{2}$ with $-t^{2}$ !

\subsection{Analytic expressions for the example}

In fact, the electrical potential anywhere in the dielectric medium is

$$
\begin{gathered}
v\left(x, z ; t_{0}\right)=\mathcal{F}_{k \rightarrow x}^{-1}\left\{\mathrm{e}^{-k\left(t_{0}+z\right)}\right\}-\frac{1}{\pi x} \int_{0}^{\infty} \frac{\cosh \left(k\left(t_{0}+z\right)\right) \sin (k x)}{k} \mathrm{~d} k \\
-\frac{\mathrm{i}}{\pi x} \int_{0}^{\infty} \frac{\sinh \left(k\left(t_{0}+z\right)\right) \cos (k x)}{k} \mathrm{~d} k .
\end{gathered}
$$


The reader may verify that (5.6) satisfies (2.2) and boundary condition (5.1) (note the sign difference between second derivatives of circular and hyperbolic trigonometric functions).

Similary, the field anywhere in the time-varying scattering medium is

$$
u\left(z, t ; t_{0}\right)=\delta\left(t-z-t_{0}\right)-\frac{1}{t} 1\left(t-z-t_{0}\right) .
$$

Again, the reader may verify that (5.7) satisfies (4.1). However, there is an important point worth noting here. Equating orders of singularity (justified in [9]) shows in general that

$$
U\left(z, z+t_{0}\right)=-2\left(\frac{\partial}{\partial z}+\frac{\partial}{\partial t}\right) \tilde{u}\left(z, t=z+t_{0} ; t_{0}\right)
$$

where $\tilde{u}\left(z, t ; t_{0}\right)$ multiplies $1\left(t-z-t_{0}\right)$. Comparing (5.8) to the continuous limit of (4.5) shows that there is a factor of two present in (5.8) that is not present in (4.5). This factor of two must be used if the problem is a discretization of a continuous problem with an impulse. This was noted below (4.5).

\subsection{Results of numerical implementation for the example}

Due to the various singularities, e.g., $1 / t$, it is not at all evident that a discretized numerical implementation of our proposed algorithm applied to this particular example will work. However, in fact it does. Using $\Delta=0.001$, a Matlab program produced numerical results virtually identical to the analytic expressions. A slight numerical error was observed near the singularities, but it had little effect on the numerical operation of the algorithm, since the percentage error was very small.

Since the discretization $V_{n}(i)$ of the potential $U(z, t)$ (see above (4.4)) is the direct output of the algorithm, we compare in figure 1 the computed potential $V_{n}(i) / \Delta^{2}$ as a function of time $t=i \Delta$ with the actual potential $U(z, t)=-2 / t^{2}$. Note that the agreement is almost perfect. Virtually identical results were obtained at all depths, as expected.

\subsection{Born approximation for the example}

The Born approximation is a linearization of an inherently nonlinear inverse problem. It has the obvious advantage of being much easier to implement, but neglecting the effects of the nonlinearities can produce significant errors.

For this example, we implement the Born approximation as follows. The integral transformation is linear, so it is unaltered. The nonlinearities occur in the propagation of the layer-stripping algorithm (4.3), in the product term $V_{n}(i) u_{n-1}(i, j)$. Neglecting this term is equivalent to neglecting multiple scattering in the time-varying inverse scattering problem. In the algorithm, this is equivalent to propagating $u_{n}(i, j)$ using just the left side of (4.3). The algorithm then computes the scattered wavefield at depth $z=n \Delta$ as simply the scattered wavefield at the surface advanced in time by $n \Delta$. Note that this is equivalent to the seismic technique of 'migration' of the scattered wavefield.

Analytically, this yields (compare carefully to (5.7))

$$
u\left(z, t ; t_{0}\right)=\delta\left(t-z-t_{0}\right)-\frac{1}{t+z} 1\left(t-z-t_{0}\right) .
$$

Application of (5.8) (which is linear, so it is unaltered) to (5.9) gives

$$
U\left(z, z+t_{0}\right)=-4 /\left.(t+z)^{2}\right|_{t=z+t_{0}}=-4 /\left(2 z+t_{0}\right)^{2} \neq-2 /\left(z+t_{0}\right)^{2},
$$




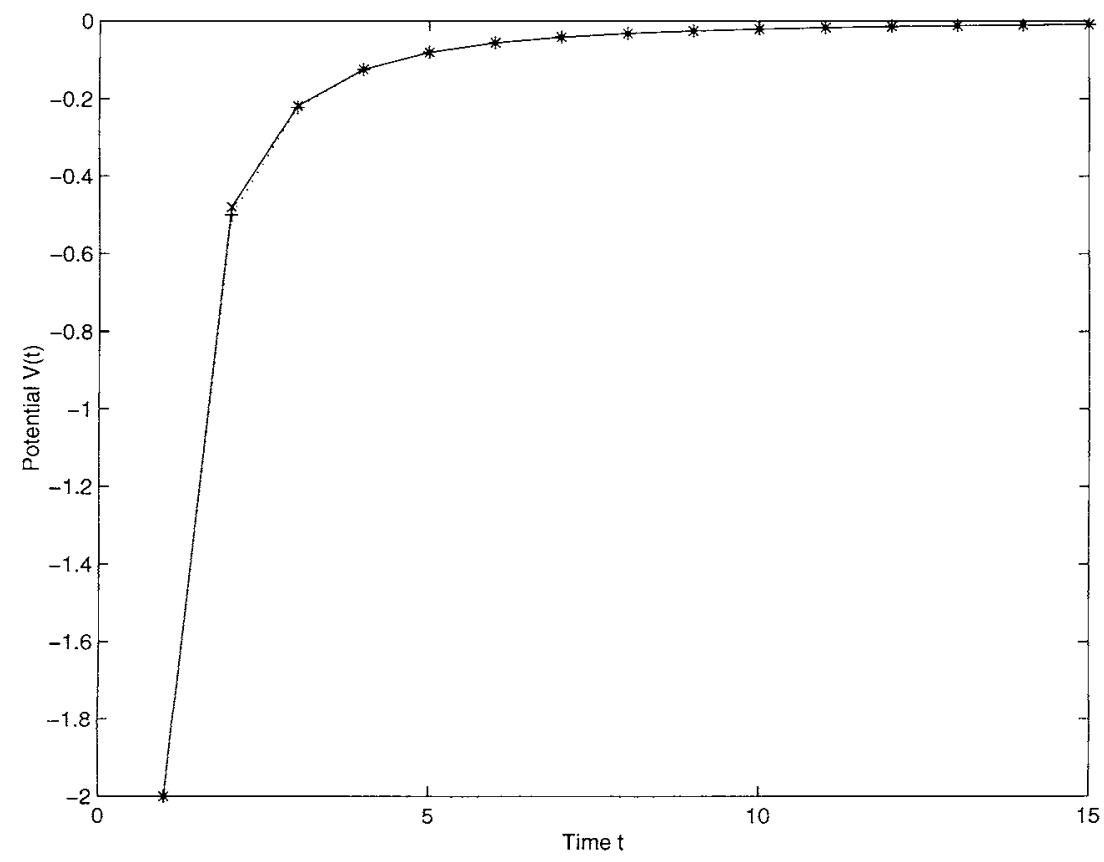

Figure 1. Actual $(+)$ and reconstructed $(\times)$ potentials.

which is clearly the wrong answer. Note that the error increases with depth $z$; this is as expected, since the amount of multiple scattering neglected increases with depth $z$. For comparison, note that (5.8) applied to the actual scattered field in (5.7) results in

$$
U\left(z, z+t_{0}\right)=-2 /\left.t^{2}\right|_{t=z+t_{0}}=-2 /\left(z+t_{0}\right)^{2},
$$

which is of course the correct answer (see (5.5)).

\section{Conclusion}

We have formulated and solved the 2D inverse conductivity problem using a new transformation that transforms the problem into a time-varying $1 \mathrm{D}$ inverse scattering problem. The results of this paper differ from previous work (including our own) on applying layer stripping to inverse conductivity problems in that no measurements were required along a medium-invariant axis, and any ill conditioning of the problem was collected at the beginning in the form of the linear transformation. Once this is done, layer stripping may be used to solve a well posed problem, so that it should be numerically stable.

We have also analysed for the first time the time-varying Schrödinger equation inverse scattering problem. We derived a layer-stripping algorithm, a discrete time-varying generalization of the Gel'fand-Levitan integral equation, and a feasibility condition on the reflection response computed from the transform. This latter condition allows this response to be corrected to ensure that the layer-stripping algorithm or nested systems of equations are solving a realistic problem, so that these techniques should be numerically stable.

We have also presented a simple but illustrative analytic example that demonstrates how our proposed approach works. This example was also implemented numerically to demonstrate that the various quantities can in fact be accurately computed. This was not trivial, due to the 
presence of singularities in $U(x, z)$ and $v(x, z)$, but the numerical results confirmed that the method works.

Future work to be performed includes (i) a detailed study of the effect of noise on the operation of the algorithm, (ii) the proper regularization technique to be used on the integral transformation or inverse Laplace transform [7] and (iii) a detailed study of how the feasibility condition helps. The results of [13] for layer-stripping algorithms for the 2D inverse scattering problem, in which a similar feasibility condition was shown to stabilize layer-stripping algorithms applied to noisy data, are very suggestive and encouraging in this respect.

\section{Acknowledgment}

This research was supported by the Office of Naval Research under grant no N00014-94-10519 .

\section{References}

[1] Pekeris C L 1940 Direct method of interpretation in resistivity prospecting Geophysics 5 31-42

[2] Koefoed O 1970 A fast method for determining the layer distribution from the raised kernel function in geoelectrical sounding Geophys. Prospect. 18 564-70

[3] Coen S and Yu M W-H 1981 The inverse problem of the direct current conductivity profile of a layered earth Geophysics 46 1702-13

[4] Levy B C 1985 Layer-by-layer reconstruction methods for the earth resistivity from direct current measurements IEEE Trans. Geosci. Remote Sens. 23 841-50

[5] Yagle A E 1987 A layer stripping fast algorithm for the two-dimensional direct current inverse resistivity problem IEEE Trans. Geosci. Remote Sens. 25 558-63

[6] Weidelt P 1972 The inverse problem of geomagnetic induction Z. Geophys. 38 257-89

[7] Krylov V I and Skoblya N S 1969 Handbook of Numerical Inversion of Laplace Transforms (Jerusalem: Israel Program for Scientific Translations)

[8] Isaacson D and Cheney M 1990 Current problems in impedance imaging Inverse Problems in Partial Differential Equations ed D Colton, R Ewing and W Rundell (Philadelphia, PA: SIAM)

[9] Bruckstein A M, Levy B C and Kailath T 1985 Differential methods in inverse scattering SIAM J. Appl. Math. $45312-35$

[10] Yagle A E 1997 Inverse scattering for time-varying one-dimensional layered media: algorithms and applications Inverse Problems 13 867-81

[11] Hsue J-J and Yagle A E 1994 Blind deconvolution of symmetric noncausal impulse responses using two-sided linear prediction IEEE Trans. Signal Process. 42 1509-18

[12] Kunetz G and d'Erceville I 1962 Sur certaine propriétés d'une onde acoustique plane de compression dans un milieu stratifié Ann. Géophys. 18 351-9

[13] Yagle A E and Frolik J 1996 On the feasibility of impulse reflection response data for the two-dimensional inverse scattering problem IEEE Trans. Antennas Propagat. 44 1551-64

[14] Yagle A E and Raadhakrishnan P 1992 Numerical performance of layer stripping algorithms for two-dimensional inverse scattering problems Inverse Problems 8 645-65

[15] Bube K P and Burridge R 1983 The one-dimensional inverse problem of reflection seismology SIAM Rev. 25 497-559 